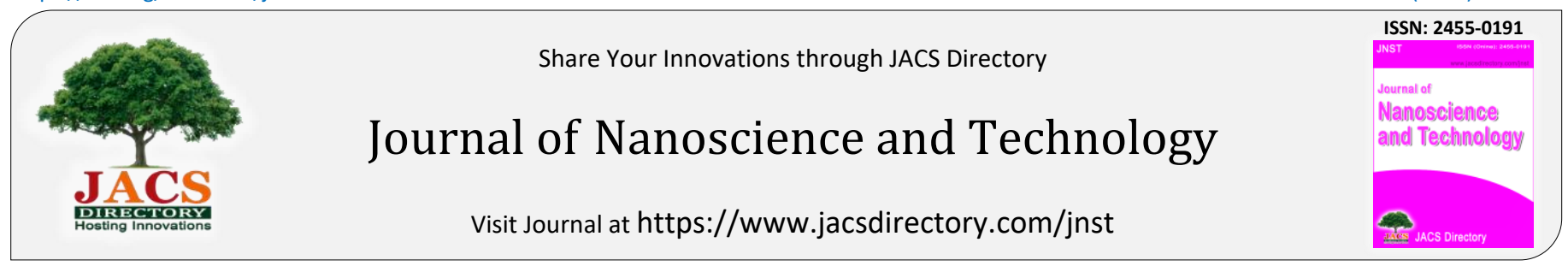

\title{
Biomediated Green Synthesis of Copper Nanoparticles using Piper nigrum Leaf Extract
}

\author{
C. Sreeja ${ }^{1}$, K. Annieta Philip ${ }^{1,}{ }^{*}$, K. Shamil2 ${ }^{2}$, O.P. Asraj ${ }^{2}$, S. Sreeja ${ }^{2}$ \\ ${ }^{1}$ Department of Physics, The Cochin College, Kochi - 682 002, Kerala, India. \\ ${ }^{2}$ Departments of Physics, Al Ameen College, Edathala - 683 564, Kerala, India.
}

\section{ART I C LEDETAILS}

\section{Article history:}

Received 30 June 2020

Accepted 31 July 2020

Available online 14 September 2020

\section{Keywords:}

Green Synthesis

Pepper Leaf Extract

Copper Nanoparticles

\begin{abstract}
A B S T R A C T
In the present days the development of efficient green synthesis of metal nanoparticles has become a major focus of researchers. It was aimed in order to find a safe, economic and eco-friendly technique for the production of well characterized nanoparticles. The present study reports the green synthesis of copper nanoparticles using plant extract (pepper leaf extract) and a copper salt (copper chloride, $\mathrm{CuCl}_{2}$ ). Copper nanoparticles are produced by the reduction of $\mathrm{CuCl}_{2}$, while the aqueous leaf extract act as reducing agent. The formation of copper nanoparticles was recognized by the change of colour from blue to black. The synthesized nanoparticles were then characterized through EDAX, XRD, and UV-Vis spectrophotometry. EDAX confirmed the formation of copper nanoparticles. The UV-Vis spectrophotometer analysis confirmed the absorption peak of copper nanoparticles at wavelength of 500-700 $\mathrm{nm}$. XRD analysis revealed three sharp peaks specifically referred to face centered cubic structure of metallic copper. This work was also carried out the optical studies of copper nanoparticles which were exposed to direct sunlight. This study reflected the effect of sunlight intensity on the absorption peak of copper nanoparticles.
\end{abstract}

\section{Introduction}

Nanotechnology and nanoparticles have been an area of attentiveness since few decades. Among the various nanoparticles metal nanoparticles have gained special importance since they are cheaper and easy to synthesis. Metal nanoparticles have been majorly focused by investigators due to their unique optical, mechanical, magnetic, electrical and chemical properties [1-3]. Several methods like mechanical milling and chemical reduction have been used to synthesis nanoparticles over the years. Now the researches take effort to improve certain green trials for the synthesis of nanoparticles. Several nanoparticles like $\mathrm{ZnO}$, gold, iron, silver etc., have been synthesized using plant extracts [4-8]. The synthesis of metal nanoparticles using plant extracts has technologically advanced a cost effective and rapid bio-synthetic protocol for the bulk synthesis of metallic nanoparticles. More research is being done to synthesis nanoparticles through green routes as it can play a chief part in the upgrading of nanotechnology and make easy to get the profits of these products for the society and environment. Copper is nowadays preferred by the researchers due to high conductivity and good potential reduction $[9,10]$.

Copper is a high strength metal and alloy plays an important role in the field of electricity. The use of copper $(\mathrm{Cu})$ and copper-based nanoparticles have caused a great deal of interest in this decade specifically in the field of catalysis. In this work, new method has established by green synthesis of copper nanoparticles by chemical reduction using pepper leaf extract. Piper nigrum, usually known as pepper, is a flowering climber in the family Piperaceae. Usually farmer cultivated pepper for its fruit which is used as a spice. In this work we have taken the fresh leaf of pepper to make leaf extract to produce the nano sized copper particles. The preparation of nanoparticles in the green environment is cost effective and innocuous. The prepared copper nanoparticles were exposed to direct sunlight over two days. The UV-Vis spectrophotometry (UV-Vis), XRD and EDAX analysis were carried out to characterize the produced copper nano particles.

\section{Experimental Methods}

Piper nigrum leaf was collected from Kalamassery in Ernakulam district, Kerala. The collected leaf was cleaned in running water and was

packed in a polythene bag. This was then taken to the laboratory and was washed thoroughly with distilled water several times. The leaf was made into small bits. $10 \mathrm{~g}$ of leaf was taken and $100 \mathrm{~mL}$ distilled water was added to it and was allowed to boil for 15 minutes. Then allowed to cool and then filtered out through Whatman No.1 filter paper. The filtrate was taken for further experimental use. $10 \mathrm{~mL}$ of $0.1 \mathrm{M}$ copper chloride was taken and $50 \mathrm{~mL}$ of pepper leaf extract was added on constant stirring. The $\mathrm{pH}$ of the solution was adjusted to alkaline by adding $5 \%$ solution of $\mathrm{NaOH}$. This was kept in magnetic stirrer for 3 hours in $100{ }^{\circ} \mathrm{C}$ by constant stirring. We can note the change of color from blue to black. The extract was kept overnight in room temperature at a dark place, and was filtered the next day using Whatman No.1 filter paper. The precipitate was taken and dried in the microwave oven at $40{ }^{\circ} \mathrm{C}$ for two days. The dried precipitate was then grinded using mortar and pestle and was made into fine powder (Sample 1). The entire experiment was repeated and the final precipitate was kept in direct sunlight for two days for drying. The average temperatures of those two days were $32{ }^{\circ} \mathrm{C}$. This dried precipitate was also made into fine powder (Sample 2). Both the samples were then characterized by XRD, EDAX and UV-Vis spectrophotometer.

(a)

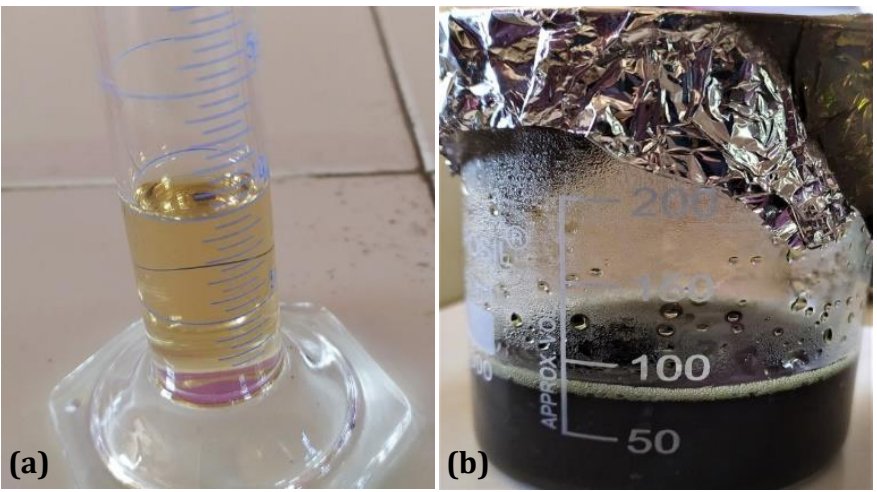

Fig. 1 (a) Pepper leaf extract and (b) formation copper nanoparticles

The structural characterizations were done by using XRD analysis and EDAX spectrum. Band gap of the prepared metal nanoparticles was calculated by using UV-Vis absorption spectrum. 


\section{Results and Discussion}

\subsection{X-Ray Diffraction Study}

The synthesized nanoparticles (sample 1) was characterised by using XRD pattern was done by using Bruker D8 Advance device. Fig. 2 shows the XRD pattern of synthesized copper nanoparticles using pepper leaf extract. The pattern obtained from the XRD of synthesized nanoparticles was analysed and it confirm the crystallinity of the copper nanoparticles. The intensity of the peak was very high compared to the reported work [11]. XRD pattern displays $\mathrm{Cu}$ characteristic diffraction peaks at around $2 \theta$ $=42^{\circ}, 49.8^{\circ}, 74^{\circ}$ corresponding to (111), (200), (220) planes of FCC Cu crystals (JCPDS No. 04-0836). The size of formed nanoparticles calculated from Debye Scherer's formula was 13-18 nm.

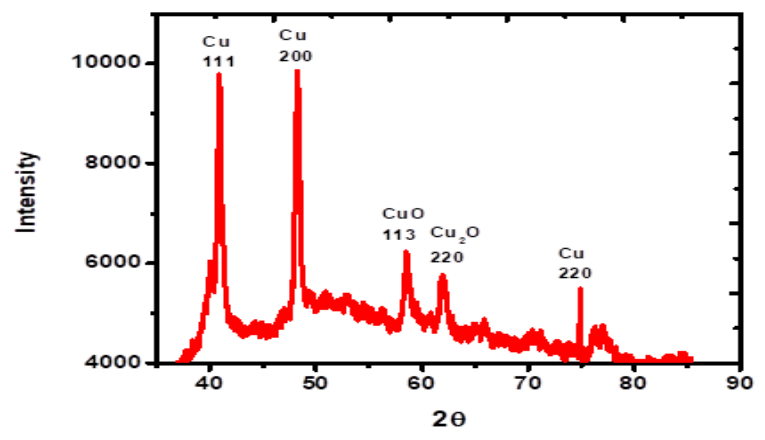

Fig. 2 XRD pattern of copper nanoparticle

\subsection{EDAX Spectral Study}

Energy dispersive X-ray analysis (EDAX) is an analytical technique used for the elemental analysis or chemical characterization of a sample. EDAX was performed with Jeol 6390LA/ OXFORD XMX N instrument. The EDAX spectrum is presented as Fig. 3. The spectrum indicates the presence of $\mathrm{Cu}$ and small amount of oxygen. The spectrum shows the formation of pure copper (47.6\%) in copper nanoparticles.
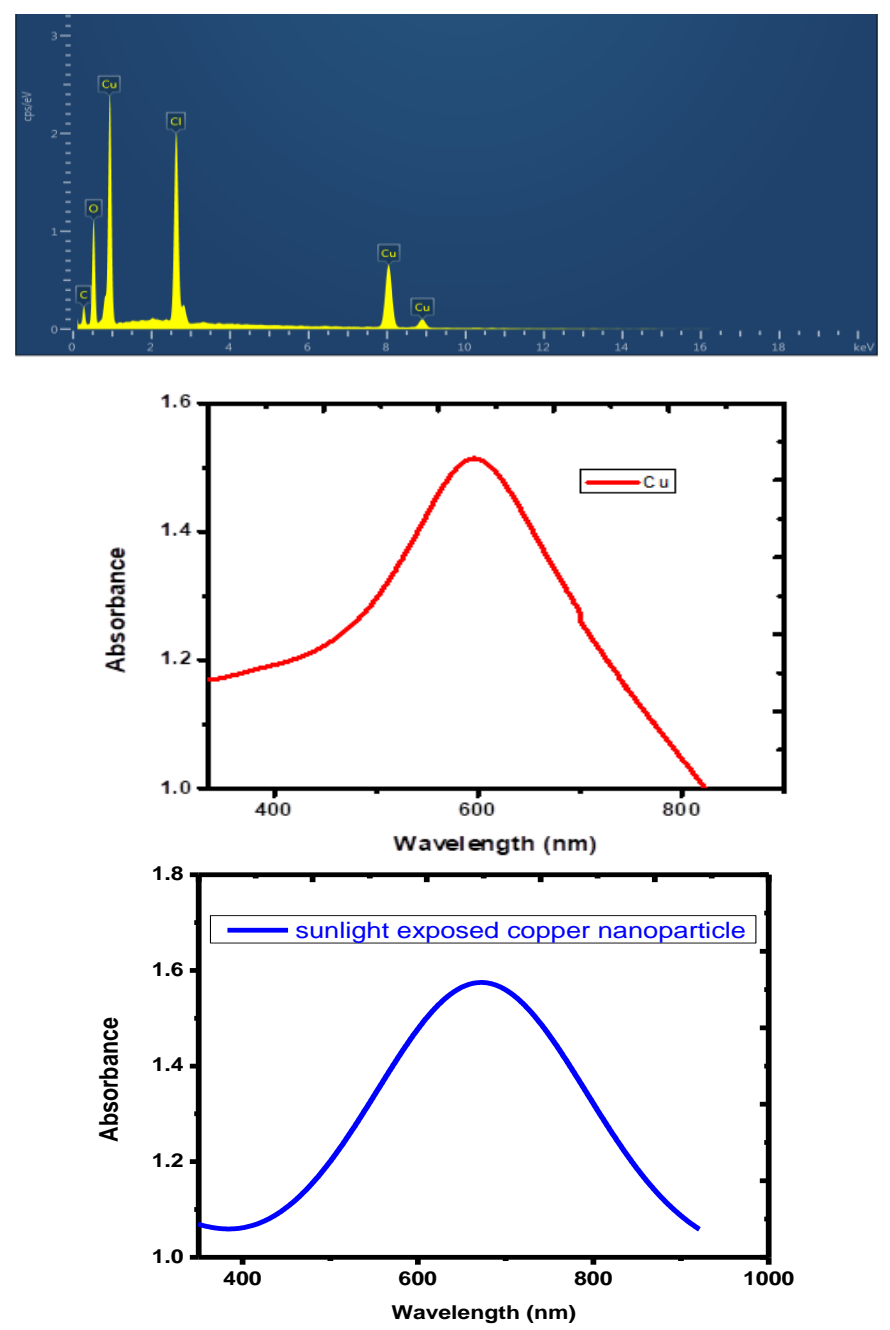

Fig. 4 UV-Vis absorption spectrum of copper nanoparticles https://doi.org/10.30799/jnst.305.20060303

\subsection{UV-Visible Absorption Study}

UV -Vis spectra of copper nanoparticles was done using UV-Vis NIR spectrophotometer Agilent Cary $5000200 \mathrm{~nm}$ to $3000 \mathrm{~nm}$ DRS integrating sphere diameter $150 \mathrm{~mm}$, angle of incidence $8^{\circ}$. Fig. 4 shows the UV-Vis absorption spectra of prepared copper nanoparticle and sunlight exposed copper nanoparticles. The spectrum shows a characteristic absorption peak at around $500-700 \mathrm{~nm}$. The sharp peak properties indicate that the formed particles are in nano sized range. The peak formed in the spectrum is due to surface plasmon resonance absorption of copper nanoparticles. The absorption spectrum of sunlight exposed copper nanoparticle was also studied. It can be seen that the maximum absorption peak was red shifted from $596 \mathrm{~nm}$ to $671 \mathrm{~nm}$ in the presence of sunlight. Comparing the UV-Vis spectra given in Fig. 4, it can be confirmed that the optical properties of formed copper particle were affected by the direct sunlight. The band gap of copper nano particles was calculated from the absorption spectrum using Tauc's plot (Fig. 5) using the equation, $\alpha h \gamma=D(h \gamma-E g)^{\mathrm{n}}$ where $h \gamma$ the energy of the photon is, Eg is the band gap of material and D is constant. The band gap energy was calculated as $1.5 \mathrm{eV}$ and $1.41 \mathrm{eV}$ for prepared copper nanoparticles and sunlight exposed copper nanoparticles respectively.
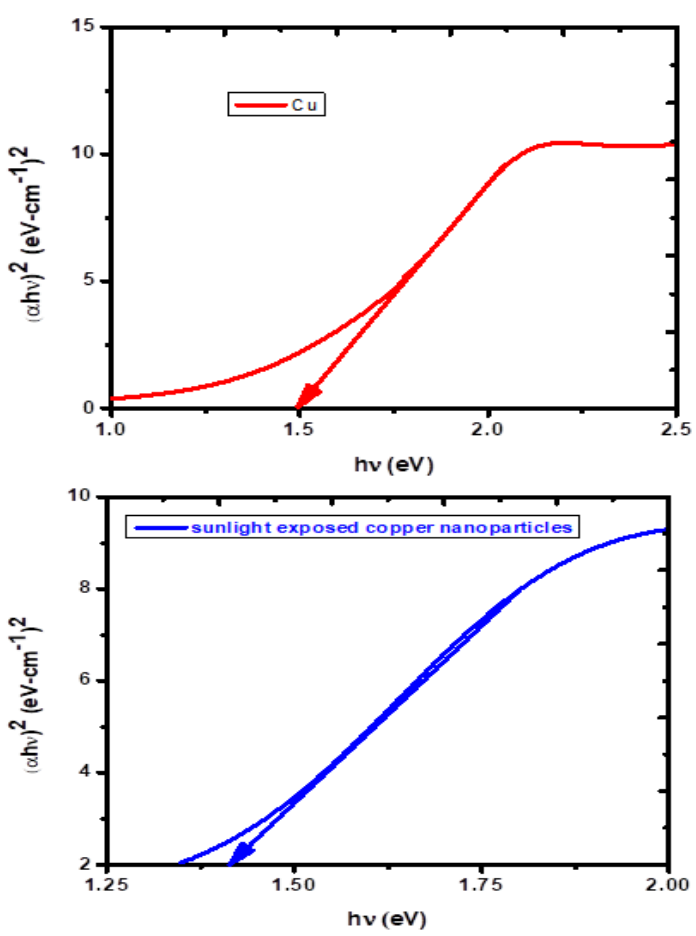

Fig. $5(\alpha h \gamma)^{2}$ vs $(h v)$ graph of copper nanoparticles

\section{Conclusion}

This present work demonstrated the formation of copper nanoparticles using a natural and low-cost reducing agent (pepper leaf extract). Copper nanoparticles can be produced by avoiding the presence of the hazardous and toxic solvents and waste. The color change of the copper chloride solution indicates the formation of copper nanoparticles. The formation of nanoparticles was further confirmed by the surface plasmon resonance peak obtained in the UV-Vis spectra. Formation of nanoparticles was again confirmed by EDAX spectrum and XRD studies. The experiment has been repeated under sunlight confirmed that the absorption peak of copper nanoparticle was affected by direct sunlight. In future studies the optical properties of copper nanoparticles will be studied by changing the duration of sunlight exposure.

\section{Acknowledgements}

The authors would like to acknowledge Instrumentation Centre (STIC), Cochin University of Science and Technology and M.G. University, Kottayam for extending their facilities to this work.

\section{References}

[1] S.C. Singh, R.K. Swarnkar, R. Gopal, Synthesis of copper/copper-oxide nanoparticles: Optical and structural characterizations, AIP Conf. Proc. 1147 (2009) 205-210. 
[2] B. Balamurugan, B.R. Mehta, S.M. Shivprasad, Surface-modified CuO layer in size-stabilized single-phase $\mathrm{Cu}_{2} \mathrm{O}$ nanoparticles, Appl. Phys. Lett. 3176 (2001) 79-84.

[3] H. Lin, C. Wang, H.C. Shih, J. Chen, C. Hsieh, Characterizing well-ordered CuO nano fibrils synthesized through gas-solid reactions, J. Appl. Phys. 95(10) (2004) 5889-5895.

[4] J.J. Zhang, J.F. Liu, Y.D. Li, Nearly monodisperse $\mathrm{Cu}_{2} \mathrm{O}$ and $\mathrm{CuO}$ nanospheres: Preparation and applications for sensitive gas sensors, Chem. Mater. 18(4) (2006) 867-871.

[5] N.A. Dhas, C.P. Raj, A. Gedanken, Synthesis, Characterization, and properties of metallic copper nanoparticles, Chem. Mater. 10(5) (1998) 1446-1452.

[6] M. Samim, N.K. Kaushik, A. Maitra, Effect of size of copper nanoparticles on its catalytic behaviour in Ullman reaction, Bull. Mater. Sci. 30 (2007) 535-540.

[7] Y. Suresh, S. Annapurna, G. Bhikshamaiah, A.K. Singh, Green luminescent copper nanoparticles, International Conference on Advances in Materials and
Manufacturing Applications, Amrita School of Engineering, Bangalore, India, 2016.

[8] Sathiraju Annapurna, Yathapu Suresh, Bojja Sreedhar, Ganghishetti Bhikshamaiah, A.K. Singh, Characterization of green synthesized copper nanoparticles stabilized by ocimum leaf extract, Mater. Res. Soc. Symp. Proc. 1704 (2014) 1-8.

[9] Yu Zhang, Pengli Zhu, Gang Li, Tao Zhao, Xianzhu Fu, et al., Facile preparation of monodisperse, impurity-free, and antioxidation copper nanoparticles on a large scale for application in conductive ink, ACS Appl. Mater. Interf. 6(1) (2014) 560-567.

[10] Xiaoqun Zhu, Bowen Wang, Feng Shi, Jun Nie, Direct, rapid, facile photochemical method for preparing copper nanoparticles and copper patterns, Langmuir 28(40) (2012) 14461-14469.

[11] T. Theivasanthi, M. Alagar, X-ray diffraction studies of copper nano powder, Arch. Phys. Res. 1(2) (2010) 112-117. 